\title{
Water maser in Southern FIR-bright galaxies: detection and interferometric follow-ups
}

\author{
G. Surcis ${ }^{* a, b, c}$ A. Tarchi, ${ }^{a, d}$ C. Henkel, ${ }^{c}$ J. Ott, ${ }^{e, f} \mathbf{J}$. Lovell, ${ }^{g}$ and P. Castangia, ${ }^{a}$ \\ a INAF-Osservatorio astronomico di Cagliari \\ Loc. Poggio dei Pini, Strada 54, 09012 Capoterra (CA), Italy \\ ${ }^{b}$ Argelander-Institut für Astronomie (Universität Bonn) \\ Auf dem Hügel 71, 53121 Bonn, Germany \\ ${ }^{c}$ Max-Planck Institut für Radioastronomie \\ Auf dem Hügel 69, 53121 Bonn, Germany \\ d INAF-Istituto di Radioastronomia \\ Via Gobetti 101, 40129 Bologna, Italy \\ e National Radio Astronomy Observatory \\ 520 Edgemont Road, Charlottesville, VA 22903, USA \\ ${ }^{f}$ California Institute of Technology \\ 1200 E California Blvd, Caltech Astronomy 105-24, Pasadena, CA 91125, USA \\ ${ }^{g}$ Department of Maths and Physics, Univerisity of Tasmania \\ Private Bag 21, Hobart, Tasmania 7001, Australia \\ E-mail: gsurciseastro.uni-bonn.de, atarchieca.astro.it, \\ p220hen@mpifr-bonn.mpg.de, jottenrao.edu, Jim. Lovell@utas.edu.au, \\ pcastang@ca.astro.it
}

\begin{abstract}
A correlation between the presence of $22 \mathrm{GHz}$ water masers and $100 \mu \mathrm{m}$ flux density was proposed by [1] as a result of a survey on a sample of all northern galaxies (Dec $>-30^{\circ}$ ) associated with IRAS FIR point sources with $100 \mu \mathrm{m}$ flux densities larger than $50 \mathrm{Jy}$. The survey provided the relevant maser detection rate of $22 \%$, which is extremely high with respect to other surveys. Therefore, we decided to complete the sample by observing all southern galaxies (with the same FIR flux constraint and Dec $<-30^{\circ}$ ) with the 70-m Tidbinbilla telescope in a search for water maser emission. We have detected two new water masers in the merging system NGC3256 and in the spiral galaxy NGC3620, respectively, yielding a detection rate consistent with the northern sample. The detections have been followed-up through spectral line interferometric observations with the ATCA.
\end{abstract}

The 9th European VLBI Network Symposium on The role of VLBI in the Golden Age for Radio Astronomy and EVN Users Meeting

September 23-26, 2008

Bologna, Italy

\footnotetext{
* Speaker.

${ }^{\dagger}$ GS is a student of the International Max Planck Research School (IMPRS) for Radio and Infrared Atronomy.

$\ddagger$ JO is a Jansky Fellow of the National Radio Astronomy Observatory. NRAO is a facility of the National Science Foundation operated under cooperative agreement by Associated Universities, Inc.
} 


\section{Introduction}

The far-infrared (FIR) emission commonly arises from dust grains heated by newly formed stars. Therefore, a sample of FIR bright galaxies is a suitable tool to find new extragalactic $\mathrm{H}_{2} \mathrm{O}$ masers, in particular those associated with star forming regions. A correlation between the presence of $\mathrm{H}_{2} \mathrm{O}$ masers and $100 \mu \mathrm{m}$ flux density was indeed reported by [1] as a result of an Effelsberg 100-m survey of all galaxies with declination $\mathrm{Dec}>-30^{\circ}$, associated with IRAS point sources showing $\mathrm{S}_{100 \mu \mathrm{m}}>50 \mathrm{Jy}$ [2]. This correlation has recently been confirmed by [3] with a sample of 41 galaxies with $30 \mathrm{Jy}<\mathrm{S}_{100 \mu \mathrm{m}}<50 \mathrm{Jy}$ and Dec $>-30^{\circ}$. Good results obtained by [1] and [3] in the northern hemisphere have motivated us to complete the FIR sample by including all southern galaxies (with declination Dec $<-30^{\circ}$ ) associated with IRAS point sources characterized by $\mathrm{S}_{100 \mu \mathrm{m}}>50 \mathrm{Jy}$.

\section{Results and Preliminary Discussion}

The list of sources was compiled using the IRAS Point Sources Catalog [2]. Eight of these sources were observed in previous works. The sources of our sub-sample were observed in the $6_{16}-5_{23}$ transition of $\mathrm{H}_{2} \mathrm{O}$ (rest frequency: $22.23508 \mathrm{GHz}$ ) with the $70 \mathrm{~m}$ Nasa Deep Space Network antenna located at Tidbinbilla (hereafter Tidbinbilla), Australia. Two new $\mathrm{H}_{2} \mathrm{O}$ masers were detected, in the galaxies NGC 3256 and NGC 3620, which were followed-up by interferometric spectral line observations at $22 \mathrm{GHz}$ with the Australia Telescope Compact Array (ATCA). Line profiles are shown in Fig. 1.

NGC 3256 is a well studied merging galaxy located at a distance of $\sim 37 \mathrm{Mpc}\left(1^{\prime \prime} \sim 180 \mathrm{pc}\right.$; $H_{0}=75 \mathrm{~km} \mathrm{~s}^{-1} \mathrm{Mpc}^{-1}$ ). Water maser emission has been found by us in August 2007. Its isotropic maser luminosity of $\sim 10 \mathrm{~L} \odot$ places the maser near the limit between water kilomasers and megamasers. In the ATCA observation, the emission is seen to originate from two separate maser spots: a northern one (labelled $\mathrm{N}$ ) and a southern one (labelled S). Both features are redshifted with respect to the systemic velocity and their luminosities are $1.0 \mathrm{~L}_{\odot}(\mathrm{N})$ and $2.4 \mathrm{~L}_{\odot}(\mathrm{S})$. A few days after the ATCA observations, we observed the maser source again with Tidbinbilla providing a singledish luminosity of $\sim 13 \mathrm{~L}_{\odot}$, higher than that measured in 2007. The $\mathrm{H}_{2} \mathrm{O}$ kilomasers in NGC 3256 are associated with starburst processes present in the main gas disk of the galaxy.

NGC 3620 is a peculiar southern SB galaxy [4]. Located at a distance of $\sim 20 \mathrm{Mpc}\left(1^{\prime \prime} \sim 100 \mathrm{pc}\right.$; $H_{0}=75 \mathrm{~km} \mathrm{~s}^{-1} \mathrm{Mpc}^{-1}$ ). In February 2005, we detected a $\mathrm{H}_{2} \mathrm{O}$ maser in NGC 3620 that has been monitored until April 2008. The maser emission is composed of two main spectral features, denoted "a" and "b". They are red-shifted with respect to the systemic velocity of the galaxy $\left(1680 \mathrm{~km} \mathrm{~s}^{-1}\right)$. The flux density of the $a$ feature is smaller than the $b$ component. The total isotropic maser luminosity is $\sim 4.7 \mathrm{~L}_{\odot}$. In the more sensitive ATCA observation, the two features are confirmed and a third feature $(c)$ is detected between the two main ones. All three maser components arise from a spatially unresolved spot associated with the center of the galaxy. The total isotropic maser luminosity is $\sim 2.1 \mathrm{~L}_{\odot}$. The apparent swap of $a$ and $b$ between 2005 and 2006 and the subsequent swap-back in the following epochs may let us speculative about an anticorrelation between the maser features, possibly associated with an accretion disk like the one in the galactic star form- 

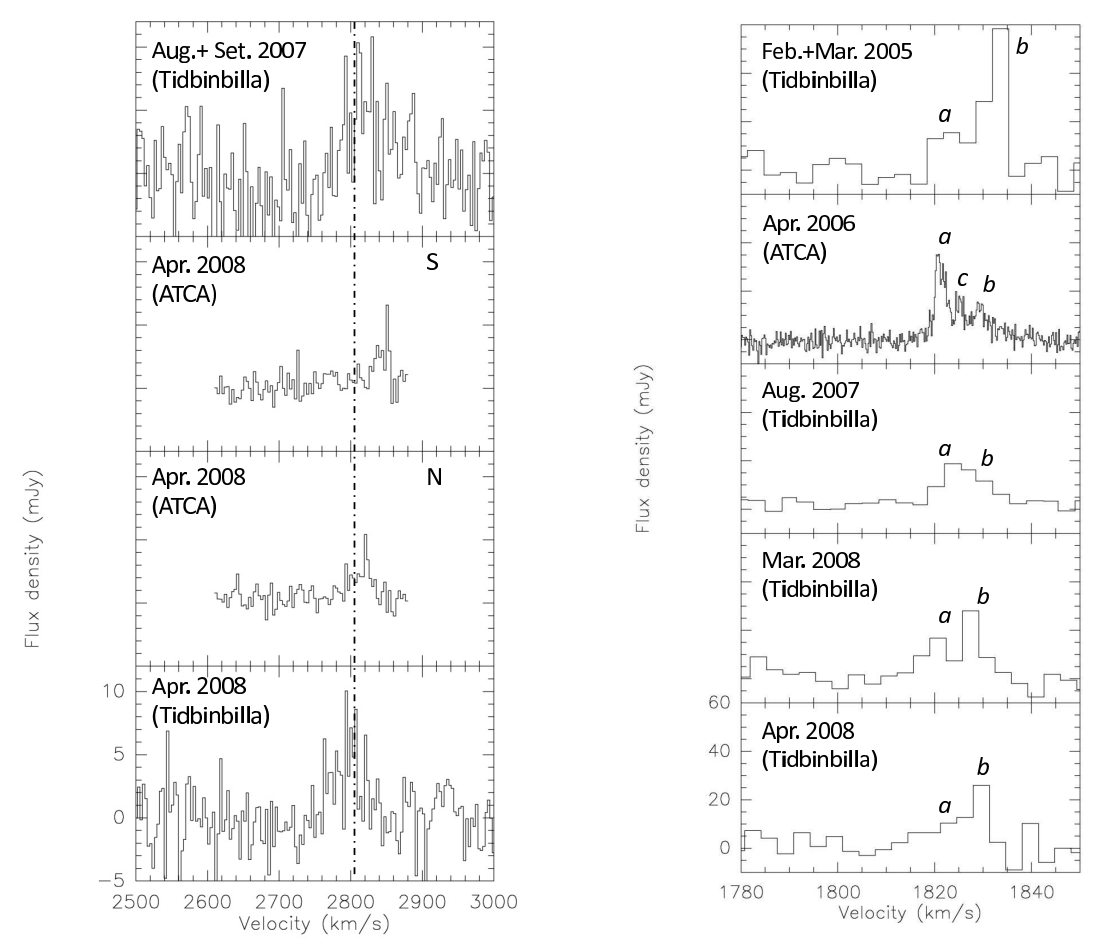

Figure 1: Left: The $\mathrm{H}_{2} \mathrm{O}$ maser in $\mathrm{NGC} 3256$. The channel spacing are $0.5 \mathrm{~km} \mathrm{~s}^{-1}$ (Tidbinbilla) and $3.7 \mathrm{~km} \mathrm{~s}^{-1}$ (ATCA), where two emission peaks have been found at different positions ( $\mathrm{N}=$ north, $\mathrm{S}=$ south).The recessional velocity of the galaxy (the dot-dashed line) is $2804 \mathrm{~km} \mathrm{~s}^{-1}$ according to the NASA/IPAC database (NED). Right: $\mathrm{H}_{2} \mathrm{O}$ spectra observed toward NGC 3620 between February 2005 and April 2008. Channel spacings are $0.5 \mathrm{~km} \mathrm{~s}^{-1}$ (Tidbinbilla) and $0.2 \mathrm{~km} \mathrm{~s}^{-1}$ (ATCA). The velocity scale for both is with respect to the local standard of rest (LSR).

ing region S255 [5]. The association of the kilomaser is still under investigation.

\section{Acknowledgments}

We wish to thank Shinji Horiuchi for performing part of the observations at Tidbinbilla and for useful discussions.

\section{References}

[1] C. Henkel, A. B. Peck, A. Tarchi, N. M. Nagar, J. A. Braatz, P. Castangia and L. Moscadelli, New $\mathrm{H}_{2} \mathrm{O}$ masers in Seyfert and FIR bright galaxies, A\&A 436 (2005) 75.

[2] L. Fullmer \& C.J. Lonsdale, Cataloged galaxies and quasars observed in the IRAS survey, Version 2, JPL D-1932 (1989).

[3] P. Castangia, A. Tarchi, C. Henkel and K.M. Menten, New $\mathrm{H}_{2} \mathrm{O}$ masers in Seyfert and FIR bright galaxies II. The intermediate luminosity range, A\&A 479 (2008) 111.

[4] T. Elfhag, R.S. Booth, B. Hoeglund, L.E.B. Johansson and A. Sandqvist, A CO survey of galaxies with the SEST and the 20-m Onsala telescope, A\&AS 115 (1996) 439.

[5] R. Cesaroni, Water maser variability and disk structure in S 255, A\&A 233 (1990) 513. 